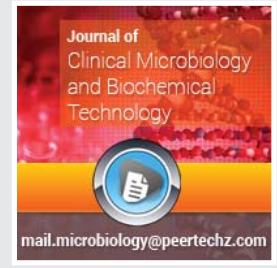

\title{
Detection of giardia lamblia genotypes in sewage and in stool samples in Israel
}

\author{
Abidelfatah M Nasser ${ }^{1 *}$, Neta-Lee Benisti ${ }^{1,2}$, Marina Taran- \\ Benshoshan ${ }^{1,2}$, Valeria Kravitz ${ }^{1}$ and Yeshayahu Nitzan² \\ 'Water Quality Research Laboratory, Ministry of Health, Israel \\ ${ }^{2}$ Faculty of Life Sciences, Bar-IIlan University, Ramat Gan, Israel
}

Received: 10 June, 2020

Accepted: 22 June, 2020

Published: 23 June, 2020

*Corresponding author: Abidelfatah M. Nasser, Water Quality Research Laboratory, Ministry of Health, Tel-Aviv, Israel, Tel: +972-03-5158682; Fax: +972-036826996; E-mail: abid.nasser@phlta.health.gov.il

Keywords: Giardia; Wastewater; Stool samples; Assemblages $\mathrm{A}$ and $\mathrm{B}$

https://www.peertechz.com

\section{Check for updates}

\begin{abstract}
Giardia lamblia is a protozoan parasite which causes a severe disease called Giardiasis. This study was conducted to determine the prevalence of Giardia cysts in raw wastewater and its prevalence in the study community. Furthermore, the prevalence of assemblages A and B in sewage was compared with their prevalence in stool samples tested positive for Giardia. All wastewater samples were found positive for Giardia at a concentration range of 10-12225 cysts/L. Positive stool samples contained Giardia at an average concentration of $1.4 \times 10^{5} \mathrm{cysts} / \mathrm{gr}$. Giardia assemblage A alone was detected in $38.2 \%$ of wastewater samples, whereas assemblage B was not detected separately. However, $61.8 \%$ of the samples were found to contain both assemblages. In stool samples, the majority 27 out of 50 ( $54 \%$ ) were found to contain assemblage B, $34.6 \%$ contained assemblage A and only $11.5 \%$ contained a mix of both assemblages. The results of the study indicate that Giardia is highly prevalent in wastewater in Israel reflecting its prevalence in the community. In addition, assemblage A is highly prevalent in wastewater, whereas assemblage B is more prevalent in stool samples, suggesting milder and/or carrier state of infection for assemblage A.
\end{abstract}

\section{Introduction}

Giardia intestinalis (also known as G. lamblia and G. duodenalis) is a waterborne protozoan parasite [1]. Giardia causes a self-limited clinical illness (giardiasis), typically characterized by diarrhea, often associated with dehydration, bloating, weight loss, abdominal pain, nausea, malabsorption and vomiting; asymptomatic infection also occurs frequently [2]. The transmission of the protozoan parasites occurs mainly through the fecal-oral route, person to person or by exposure to contaminated water and food [3-5]. The infectious dose is low; ingestion of 10 cysts has been reported to cause infection in humans (Rendtorff 1954) [6]. Infected persons have been reported to shed $10^{8}-10^{9} \mathrm{cysts}$ per day and excrete cysts for months [6].

The prevalence and concentration of Giardia cysts in raw wastewater depend on several factors such as sanitation conditions, socioeconomic status, levels of annual precipitation and on the sensitivity of the detection methods. Giardia cysts have been reported to be prevalent at higher levels in raw wastewater samples analyzed in various parts of the world. Giardia cysts were detected in almost $100 \%$ of the analyzed raw wastewater samples in various countries at a concentration range of 2 to 13,600 cysts/L and no substantial differences were observed in the concentration of Giardia cysts in raw wastewater from various countries [7].

A considerable amount of data has shown that Giardia should be considered as a species complex that comprises at least eight distinct genetic groups, referred to assemblages A to $\mathrm{H}[8-10]$. To date, only assemblages $\mathrm{A}$ and $\mathrm{B}$ have been associated with human infections. Genotypes $\mathrm{C}$ to $\mathrm{H}$ are known as pathogens of animals. However, genetic traits that influence the virulence and other aspects of the infection are unknown and efforts to correlate the parasite genetic make-up and the clinical symptoms in the host have generated controversial results $[11,12]$. This study was performed to determine the prevalence and concentration of Giardia assemblages in Giardia positive stool in comparison with assemblages present in wastewater. 


\section{Material and Methods}

\section{Raw wastewater and stool samples}

Forty-two raw wastewater samples of one liter were collected from two wastewater treatment plants (WWTP), plant A serves 240,000 and plant B serves 120,000 inhabitants. Grab samples were collected once a month in 2012 and 2013. Giardia cysts were concentrated from $0.2 \mathrm{~L}$ of raw wastewater samples by centrifugation for 15 minutes at $1100 x g$. Fifty coded clinical stool samples $(1 \mathrm{ml})$, positive for $\mathrm{G}$. intestinalis were obtained from Clalit Health Services Laboratories. Concentrated parasites from stool and wastewater samples were affinity purified and concentrated by Immunomagnetic Separation (IMS) using magnetic beads coated with antiGiardia monoclonal antibodies (Dynal, Lake Success, N.Y.) as previously described in method1623.1 of the USEPA [13]. At this stage, $20 \%$ of the purified sample was used for immunefluorescent staining and microscopic enumeration of Giardia cysts. In parallel, the remaining $80 \%$ of the concentrated sample (complex of immune magnetic beads and parasites) was stored at $-80^{\circ} \mathrm{C}$ for later DNA extraction for Giardia detection by PCR and genotyping. The recovery efficiency of Giardia cysts from raw wastewater samples was $49 \pm 14.5 \%$.

\section{DNA extraction from giardia cysts}

DNA was extracted from Giardia cysts bound to the immune magnetic beads without dissociation of cysts using the QIAamp DNA mini kit (QIAGEN, Valencia, Calif.) according to the manufacturer's instructions with minor modification. Briefly, $180 \mu \mathrm{L}$ ATL buffer, from the kit, was added into 1.5-mL tubes containing the beads and subjected to ten freeze-thaw cycles in liquid nitrogen. To break the cysts wall, samples were incubated in $4 \mathrm{U}^{-1}$ protease (Sigma-Aldrich, St. Louis, MO USA) for 10 minutes at $56^{\circ} \mathrm{C}$. DNA extraction from the cysts was then performed following the manufacturer-recommended protocol. After purification, a $200 \mu \mathrm{l}$ DNA sample was stored at $-80^{\circ} \mathrm{C}$ for later analysis.

\section{PCR amplification}

Extracted Giardia DNA from stool or wastewater samples was subjected to PCR amplification of $\beta$-Giardin gene fragment of 759 and/or a 384 bp fragment as described by Caccio, et al. 2002 [14]. These fragments were utilized to differentiate assemblage A and assemblage B as determined by PCR-RFLP. PCR was conducted as previously described by Caccio, et al. 2002 [14] and Bovine Serum Albumin) BSA) was added to the PCR mixture at a concentration of $400 \mathrm{ng} / \mu \mathrm{L}$ to prevent inhibition of the DNA-polymerase activity. The PCR products were visualized by $1.5 \%$ agarose gel electrophoresis and staining with ethidium bromide under UV irradiation.

\section{RFLP - Restriction Fragment Length Polymorphism ana- lysis}

The PCR products were subjected to digestion by restriction enzyme HaeIII (FastDigest HaeIII, Thermo Scientific) as previously described [15]. This restriction enzyme produces specific fragments which enable the classification of the detected species as assemblages A and B. Restriction enzyme HaeIII produces the following fragment sizes (bp) 202, 201,
150,126, 70, 34, 4 with Giardia duodenalis Assemblage A and 202, 176, 150, 117, 84, 34, 24 Giardia duodenalis Assemblage B (Figure 1). The results of some samples were a mixture of the fragments for assemblage A and B, as shown in Figure 1.

\section{Results}

Forty two raw wastewater samples from two treatment plants were analyzed and all $(100 \%)$ samples were found positive for Giardia cysts. The concentration of Giardia cysts in raw wastewater ranged between10 to 12,225 cysts/L and the average concentration was 3685 cysts/L (Table 1).

In stool samples positive for Giardia, assemblage B was dominant and was detected in 27 out of 50 (53.85\%) samples (Figure 2). Assemblage A was less prevalent than assemblage B in Giardia positive stool samples and was detected in 17 out of $50(34.62 \%)$ samples. Assemblage A and B mixture was detected in 6 out of $50(12 \%)$ of the stool samples.

Figure 3 presents the results of the relationship between the Giardia assemblage present in the stool samples and the level of Giardia cysts detected. The majority (71.4\%) of stool samples of assemblage B contained Giardia at a concentration range of $10^{5}$ to $10^{6}$ cysts/gr, whereas, $66.4 \%$ of stool samples of either assemblage A or of the mixture contained Giardia at a concentration range of $10^{4}$ to $10^{5}$ cysts/gr. The results show that stool samples of assemblage B contain one to two orders of magnitude Giardia cysts greater than samples of assemblage A.

The results of genotyping of Giardia lamblia cysts present in raw wastewater have shown that in 16 out of $42(38.2 \%)$ of the raw wastewater samples assemblage A was the only genotype detected. Whereas, assemblage $\mathrm{B}$ alone was not detected in any one of the raw wastewater samples. A mixture of assemblages A and B was detected in 26 out of $42(62 \%)$ of the raw wastewater samples. The results demonstrate that assemblage A of Giardia is more prevalent than assemblage B

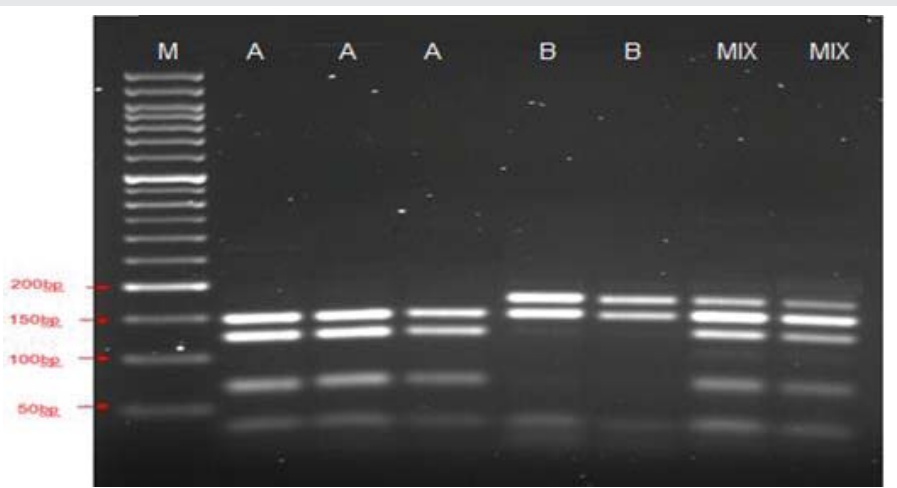

Figure 1: Restriction pattern of assemblages $A, B$ and a mix following RFLP-PCR. Assemblage A (150,126,70,34,4 bp), Assemblage B (176,150,34,24 bp) and a Mix $(176,150,126,70,34,24,4$ bp). M - Marker 50 bp.

Table 1: Prevalence and concentration of Giardia cysts in raw wastewater

\begin{tabular}{|c|c|c|}
\hline & WWTP-A & WWTP-B \\
\hline \% positives & $100(20 / 20)$ & $100(22 / 22)$ \\
\hline Avg. concentration cyst/L & 3584 & 3786 \\
\hline Mean concentration cyst/L & 2315 & 3160 \\
\hline Concentration range cyst/L & $10-11,875$ & $60-12,225$ \\
\hline
\end{tabular}

Citation: Nasser AM, Benisti NL, Benshoshan MT, Kravitz V, Nitzan Y (2020) Detection of giardia lamblia genotypes in sewage and in stool samples in Israel. J Clin Microbiol Biochem Technol 6(1): 029-032. DOI: https://dx.doi.org/10.17352/jcmbt.000040 
60
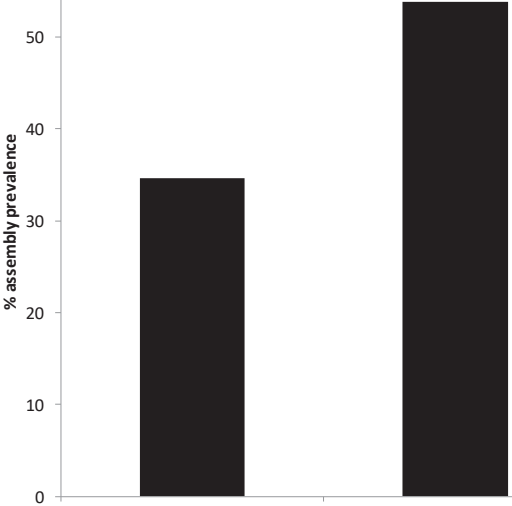

Figure 2: Prevalence of Giardia lamblia assembly A, B and a mix in Stool samples.

-10,001-100,000

$=100,001-1,000,000$

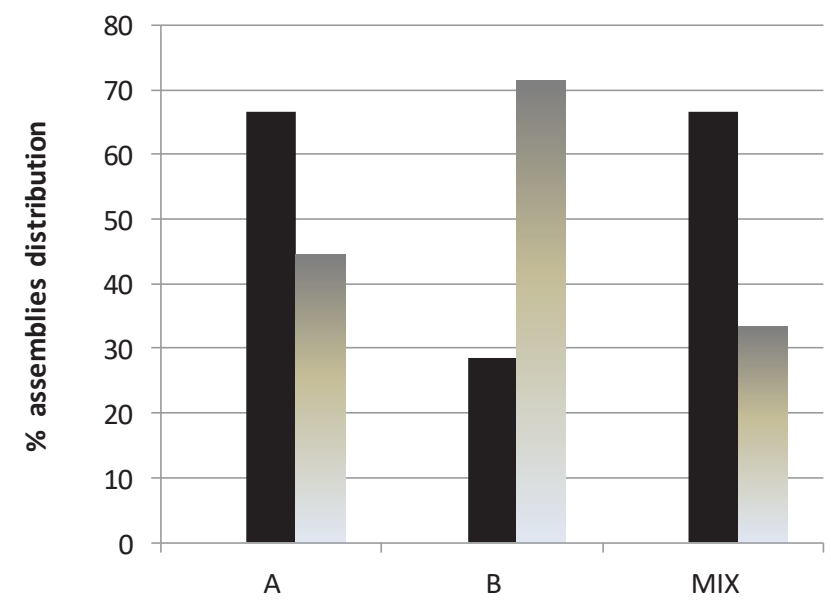

Figure 3: The relationship between the assembly present and the Giardia cyst concentration in stool samples.

in raw wastewater samples which may reflect its circulation in the community.

\section{Discussion}

All tested wastewater samples were found positive for Giardia cysts at a concentration range of 10 to 12,225 cysts/L and the mean concentration was 3160 cysts/L. The results are within the range reported previously and reviewed by Nasser, et al. 2012 [7]. The concentration range reported by Nasser, et al. 2012 [7] was up to 100,000 cysts/L with a prevalence of 50 to $100 \%$. The variation in the prevalence and levels of Giardia cysts detected in raw wastewater may be the result of sanitation conditions, wastewater quality and the sensitivity of the detection methods [7].

Assemblage B was the dominant G. duodenalis genotype detected in clinical stool samples and it was detected in $53.8 \%$ of the samples, whereas, assemblage A was detected in only $34.2 \%$. The higher prevalence of assemblage B in stool samples can stem from differences of the symptoms caused by the two genotypes. Conflicting results have been reported concerning the correlation between disease and Giardia genotype in humans. A survey conducted in Ethiopia found a significant correlation between symptomatic infection and the presence of assemblage B [16]. A similar correlation was reported by Homan \& Mank, 2001 [11], with assemblage B isolates associated with persistent diarrhea, whereas assemblage A infections were associated with intermittent diarrhea [11]. On the other hand, Sahagun, et al. 2008 [17], found a strong correlation between symptomatic infection and assemblage A2 in patients from Spain [17]. Interestingly, the proportion of asymptomatic to symptomatic infections with assemblage A was similar for three studies from Ethiopia, Bangladesh and Spain, 62\%, $57 \%$ and $67 \%$, respectively [16-18]. The key difference was that $100 \%$ of detected assemblage B infections reported from Ethiopia were associated with diarrhea, compared with $16 \%$ of infections resulting in diarrhea reported from Bangladesh and $42 \%$ from Spain [16-18]. It is expected that patients with severe diarrhea may seek medical help and stool samples may be submitted for diagnosis, whereas in cases of mild, intermittent and asymptomatic infection, the incidence may go undetected. It is worth noting that patients infected with assemblage B of G. duodenalis shed on average twice greater concentration of cysts compared with those infected with assemblage A which makes the microscopic detection easier. This can be one of the reasons for higher parentage of assemblage B detected in the stool samples.

Both Assemblages A and B were detected as mixture in $62 \%$ of the tested wastewater samples and the rest of the samples ( $38 \%$ ) contained assemblage A only. None of the wastewater samples contained assemblage $\mathrm{B}$ alone. This indicates that assemblage $\mathrm{A}$ is dominant in the studied raw wastewater. Bertrand \& Schwartzbrod [19] found that assemblages A and $B$ were detected in urban wastewater with a predominance of assembly A [19]. G. duodenalis assemblage A was detected in $92 \%$ and $100 \%$ of wastewater samples of domestic wastewater treatment plants, whereas assemblage $\mathrm{B}$ was detected in $42 \%$ and $92 \%$ of the same samples [19]. Similar results were reported by Caccio, et al. 2003 [15] who reported the presence of assemblage A in 16 out of $16(100 \%)$ wastewater samples, while assemblage B was detected in only $50 \%$ of the samples [15]. Sulaiman, et al. 2004 [20] reported the presence of G. duodenalis assemblage $A$ in $85 \%$ of raw urban wastewater samples, whereas assemblage B was detected in only $15 \%$ of the samples [20]. These results were supported by Robertson, et al. 2006 [21], who reported on the predominance of G. duodenalis assemblage $A$ in raw domestic wastewater in Norway [21]. In contrast with the results observed with urban wastewater samples, the genotyping of $G$. duodenalis cysts from human feces classified a majority of samples as assemblage B [22]. This difference between environmental and clinical samples has also been observed in other studies [21,23]. It is possible to use the levels of $G$. duodenalis in raw wastewater to estimate the circulation of the parasite in the studied community. Since giardiasis is reportable disease in many countries, it is important to evaluate the real infection rate of giardiasis based on the data established 
by wastewater monitoring. By examining wastewater samples Oda, et al. [24], estimated the prevalence of Giardia infection in inhabitants in the two study areas, in Japan to be 0.1-1.1 and $0.1-1.3 \%$ [24]. In 2010, 1956 cases of giardiasis were reported to the Department of Epidemiology of the Ministry of Health in Israel, which means. 27.9 cases of giardiasis per 100,000. However, according to the concentration of Giardia cysts in raw wastewater, the incidence was $1.6 \%$ of the population, indicating that reporting of Giardiasis cases to the Department of Epidemiology underestimates the prevalence of giardiasis in the population [7]. Giardiasis reported in the USA is lower than that reported in Israel (average 6.2 per 100,000 as compared to 27.9), these figures are also an underestimation of the number of giardiasis cases, since the mean concentration in sewage was reported to be about 5000 cysts/L [20]. Under-reporting can stem from various reasons such as: asymptomatic infection of giardiasis, low levels of excreted cysts in stool samples and the low efficiency of the reporting program.

\section{Conclusions}

The results presented demonstrate that G. lamblia is highly prevalent at high concentrations in raw wastewater in Israel. G. lamblia assemblage B is more prevalent, where 27 out of 50 (54\%), than assemblage $\mathrm{A}$ in the infected stool samples and is shed at higher concentrations. G. lamblia assemblage A is more prevalent in raw wastewater than assembly B. Reporting of Giardia incidence in the community to the Department of Epidemiology in Israel is lower than the true incidence (as estimated from the concentration in sewage) because infection can be mild or in a carrier status.

\section{References}

1. Kappus KD, Lundgren RG, Juranek DD, Roberts JM, Spencer HC (1994) Intestinal parasitism in the United States: update on a continuing problem. Am J Trop Med Hyg 50: 705-713. Link: https://bit.ly/2YmF6kf

2. Eckmann L (2003) Mucosal defenses against Giardia. Parasite Immunol 25 259-270. Link: https://bit.ly/3hSaYVg

3. Barnard RJ, Jackson GJ (1984) Giardia lamblia. The transfer of human infections by foods. In: Erlandsen, S. L., Meyer, E.A., editors. Giardia and giardiasis. New York: Plenum Press 365-377.

4. Yoder JS, Harral C, Beach MJ (2010) CDC 2010 Giardiasis surveillance United States, 2006-2008. MMWR Morb Mortal Wkly Rep 59: 15-25. Link: https://bit.ly/316bh9k

5. Birkhead G, Vogt RL (1989) Epidemiological surveillance for endemic Giardia lamblia infection in Vermont. Amer J Epidemiol 129: 762-768. Link: https://bit.ly/3fDJ9hM

6. Rendtorff RC (1954) The experimental transmission of human intestinal protozoan parasites. II. Giardia lamblia cysts given in capsules. Am J Hygiene 59: 196-208. Link: https://bit.ly/3fKyfGX

7. Nasser AM, Vaizel-Ohayon D, Aharoni A, Rebhun M (2012) Prevalence and fate of Giardia cysts in wastewater treatment plants. J Appl Microbiol 113: 477 484. Link: https://bit.ly/2Yn6DC4

8. Caccio SM, Ryan U (2008) Molecular epidemiology of giardiasis. Mol Biochem Parasitol 160: 75-80. Link: https://bit.ly/3fKyp11
9. Monis PT, Caccio SM, Thompson RC (2009) Variation in Giardia: towards a taxonomic revision of the genus. Trends Parasitol 25: 93-100. Link: https://bit.ly/2V7e3XZ

10. Xiao L, Fayer R (2008) Molecular characterization of species and genotypes of Cryptosporidium and Giardia and assessment of zoonotic transmission. Int J Parasitol 38: 1239-1255. Link: https://bit.ly/37095Ew

11. Homan WL, Mank TG (2001) Human giardiasis: genotype linked differences in clinical symptomatology. Int J Parasitol 31: 822-826. Link: https://bit.ly/37PNhbA

12. Read CM, Walters J, Robertson ID, Thompson RCA (2002) Correlation between genotype of Giardia duodenalis and diarrhoea. Int J Parasitol 32: 229-231. Link: https://bit.ly/2V75cFD

13. U.S. Environmental Protection Agency. 2005 Method 1623: Cryptosporidium and Giardia in water by IFA/IMS/FA. EPA 821-R-01-025. Office of Water, U.S. Environmental Protection Agency, Washington D.C. Link: https://bit.ly/3dIC43N

14. Caccio SM, De Giacomo M, Pozio E (2002) Sequence analysis of the B-Giardin gene and development of a polymerase chain reaction-restriction fragment length polymorphism assay to genotype Giardia duodenalis cysts from human fecal samples. Int J Parasitol 32: 1023-1030. Link: https://bit.ly/2YUcSwd

15. Caccio SM, De Giacomo M, Aulicino FA, Pozio E (2003) Giardia cysts in wastewater treatment plants in Italy. Appl Environ Microbiol 69: 3393-3398. Link: https://bit.ly/2B09Azn

16. Gelanew T, Lalle M, Hailu A, Pozio E, Cacciò SM (2007) Molecular characterization of human isolates of Giardia duodenalis from Ethiopia. Acta Trop 102: 92-99. Link: https://bit.ly/2NltjvY

17. Sahagún J, Clavel A, Goñi P, Seral C, Llorente MT, et al. (2008) Correlation between the presence of symptoms and the Giardia duodenalis genotype. Eur J Clin Microbiol Infect Dis 27: 81-83. Link: https://bit.ly/2AN5HOp

18. Haque R, Roy S, Kabir M, Stroup SE, Mondal D, et al. (2005) Giardia assemblage A infection and diarrhea in Bangladesh. J Infect Dis 192: 2171-2173. Link: https://bit.ly/3ezqXWc

19. Bertrand I, Schwartzbrod J (2007) Detection and genotyping of Giardia duodenalis in wastewater: relation between assemblages and fecal contamination origin. Water Res 41: 3675-3682. Link: https://bit.ly/2YkJQ9K

20. Sulaiman IM, Jiang J, Singh A, Xiao L (2004) Distribution of Giardia duodenalis Genotypes and Subgenotypes in raw Urban Wastewater in Milwaukee, Wisconsin. Appl Environ Microbiol 70: 3776-3780. Link: https://bit.ly/2V5RvXo

21. Robertson LJ, Hermansen L, Gjerde BK (2006) Occurrence of Cryptosporidium oocysts and Giardia cysts in sewage in Norway. Appl Environ Microbiol 72 5297-5303. Link: https://bit.ly/3drOIFE

22. Bertrand I, Albertini L, Schwartzbrod J (2005) Comparison of two target genes for detection and genotyping of Giardia lamblia in human feces by PCR and PCR-restriction fragment length polymorphism. J Clin Microbiol 43: 59405944. Link: https://bit.ly/3fJfdAP

23. Guy RA, Payment P, Ulrich JK, Horgen PA (2003) Real-Time PCR fo Quantification of Giardia and Cryptosporidium in Environmental Water Samples and Sewage. Appl Environ Microbiol 69: 95178-95185. Link: https://bit.ly/37MJLyW

24. Oda T, Kawabata M, Uga S (2005) Detection of Giardia cysts in sewage and estimations of giardiasis prevalence among inhabitants in Hyogo Prefecture, Japan. Trop Med and Health 33: 1-5. Link: https://bit.ly/3dmZBRZ

Copyright: () 2020 Nasser AM, et al. This is an open-access article distributed under the terms of the Creative Commons Attribution License, which permits unrestricted use, distribution, and reproduction in any medium, provided the original author and source are credited.

Citation: Nasser AM, Benisti NL, Benshoshan MT, Kravitz V, Nitzan Y (2020) Detection of giardia lamblia genotypes in sewage and in stool samples in Israel. J Clin Microbiol Biochem Technol 6(1): 029-032. DOI: https://dx.doi.org/10.17352/jcmbt.000040 